\title{
Plaque autofluorescence as potential diagnostic targets for oral malodor
}

\author{
Eun-Song Lee \\ Hyun-Kyung Yim \\ Hyung-Suk Lee \\ Jong-Hoon Choi \\ Ho-Keun Kwon \\ Baek-Il Kim
}




\title{
Plaque autofluorescence as potential diagnostic targets for oral malodor
}

\author{
Eun-Song Lee, ${ }^{a}$ Hyun-Kyung Yim, ${ }^{a}$ Hyung-Suk Lee, ${ }^{a}$ Jong-Hoon Choi, ${ }^{b}$ Ho-Keun Kwon, ${ }^{a}$ and Baek-II Kim ${ }^{\mathrm{a}, *}$ \\ aYonsei University College of Dentistry, Oral Science Research Institute, Department of Preventive Dentistry and Public Oral Health, \\ BK21 PLUS Project, 50 Yonsei-ro, Seoul 03722, Republic of Korea \\ bYonsei University College of Dentistry, Department of Orofacial Pain and Oral medicine, 50 Yonsei-ro, Seoul 03722, Republic of Korea
}

\begin{abstract}
The aim of this study was to determine whether the degree of tongue and interdental plaque can be used to assess oral malodor by quantifying their fluorescence as detected using quantitative light-induced fluorescence (QLF) technology. Ninety-nine subjects who complained of oral malodor were included. The level of oral malodor was quantified using the organoleptic score (OLS) and the concentration of volatile sulfur compounds (VSCs). The fluorescence properties of tongue and interdental plaque were quantified as scores calculated by multiplying the intensity and area of fluorescence in QLF-digital images, and the combined plaque fluorescence (CPF) score was obtained by summing the scores for the two regions. The associations of the scores with malodor levels and the diagnostic accuracy of the CPF score were analyzed. The two plaque fluorescence scores and their combined score differed significantly with the level of oral malodor $(p<0.001)$. The CPF score was moderately correlated with OLS $(r=0.64)$ and VSC levels $(r=0.54)$, and its area under the receiver operating characteristic curve was 0.77 for identifying subjects with definite oral malodor (OLS $\geq 2)$. In conclusion, plaque fluorescence from tongue and interdental sites as detected using QLF technology can be used to assess the level of oral malodor. $\odot 2016$ Society of Photo-Optical Instrumentation Engineers (SPIE) [DOI: 10.1117/1.JBO.21.8 .085005]
\end{abstract}

Keywords: autofluorescence; diagnosis; interdental plaque; oral malodor; tongue plaque; quantitative light-induced fluorescence technology.

Paper 160283RR received May 2, 2016; accepted for publication Aug. 9, 2016; published online Aug. 26, 2016.

\section{Introduction}

Oral malodor is primarily caused by bacteria, such as Gram-negative anaerobic and proteolytic obligate species found in the oral cavity, and their degradation of sulfur-containing amino acids into volatile sulfur compounds (VSCs) results in the observed clinical symptoms. ${ }^{1-3}$ It is well-known that the oral cavity provides favorable conditions for the growth of bacteria responsible for oral malodor. ${ }^{4-6}$ In particular, the dorsum of the tongue and the interdental areas serve as recipient sites of anaerobic bacteria, which commonly produce VSCs and short-chain organic acids and act as major sites of oral malodor generation. 3,7

The plaque that accumulates in these sites is associated with the oral malodor and the status of plaque influences the severity of the malodor and also acts as an indicator of the oral hygiene status. A strong correlation has been reported between the tonguecoating status and oral malodor, ${ }^{3,8,9}$ which highlights the need for a method that can objectively evaluate the tongue-coating status to aid the diagnosis and assessment of oral malodor. ${ }^{10}$ Given that the interdental plaque has been shown to consist of oral anaerobic organisms, especially the gingival sulcus and periodontal pockets where plaque is present produce high concentrations of VSCs and are correlated with oral malodor. ${ }^{11}$ Therefore, bacterial biofilms formed on the tongue and interdental region including the subgingival area influence the production of oral malodor as major etiological factors. $^{12,13}$

Various methods for quantifying biofilm level have been proposed for evaluations of oral malodor. These methods have been largely based on visual inspection, bacterial counts, and wet-weight measurements of the biofilm. ${ }^{14-16}$ Although visual assessments are predominantly used in clinical applications, they lack reproducibility and objectivity. It has been shown that the opacity of a biofilm can vary with the species constituting the biofilm, and it is difficult to quantify qualitative characteristics related to the pathogenicity of oral malodor. ${ }^{17}$

Quantitative light-induced fluorescence (QLF) technology has been used for detecting dental plaque as red fluorescence (RF) that originates from specific bacterial metabolites formed in an oral biofilm, such as endogenous metal-free fluorescent porphyrin, ${ }^{18,19}$ when irradiated with $405 \mathrm{~nm}$ of visible bluelight. This optical phenomenon of plaque can be explained since porphyrin compounds produced by oral microorganisms show strong fluorescence in the red spectral region when excited with violet-light ranging from 400 to $420 \mathrm{~nm}^{20,21}$ The concentration of the porphyrin compounds is reported to be high in Gram-negative oral bacteria, which increases as the dental biofilm becomes more mature. ${ }^{21-23}$ Previous studies have found that mature biofilm exhibits RF stronger than initial biofilm, and the RF intensity increases with the biofilm maturation and its cariogenicity. ${ }^{21,23-25}$ From recent studies, it has been confirmed that a plaque assessment method based on autofluorescence of oral biofilms can be used to quantify dental plaque and tongue plaque by assessing their RF intensity detected by QLF systems ${ }^{26}$ and that tongue plaque produces RF that shows a higher-than-moderate correlation with the level of oral malodor. ${ }^{27}$ If QLF technology can be used to detect bacterial biofilms that mainly comprise anaerobes producing malodorous 
compounds and to quantify their accumulation status comprehensively, this will make it possible to evaluate oral malodor more precisely than when assessing only a single-bacterial factor.

Therefore, this clinical study examined the fluorescence of tongue and interdental plaque, which are the predominant causes of oral malodor, using QLF technology and assessed the ability of the combined plaque fluorescence (CPF) score to quantify the level of oral malodor.

\section{Materials and Methods}

\subsection{Study Population}

The present cross-sectional study was carried out with 99 subjects who reported oral malodor. The study protocol was approved by Yonsei University Institutional Review Board (2-2012-0007) and followed the strengthening the reporting of observational studies in epidemiology (STROBE) guidelines. The one-sided $95 \%$ formulation of the lower confidence limit was used for sample size calculations, according to the recommendation of Flahault et al. ${ }^{28} \mathrm{~A}$ sensitivity of 0.8 and minimal acceptable lower confidence interval limit of 0.65 were expected, and the minimum sample size of 98 participants was established. ${ }^{28}$ Subjects were excluded if they had one of the following criteria: systemic disease, no intake of systemic antimicrobials during the previous 3 months, being pregnant, or currently smoking. Patients with possible extraoral causes of oral malodor such as nasal and pharyngeal infection, respiratory conditions, gastrointestinal conditions, and metabolic conditions were also excluded. Before performing the examinations, all subjects refrained for $4 \mathrm{~h}$ from consuming breakfast as well as any strong-smelling foods that might induce oral malodor and from performing any oral hygiene practices such as brushing, use of oral rinses, or chewing gum. Measurements were made between 10:00 a.m. and 12:00 p.m.

\subsection{Questionnaire}

All subjects completed a questionnaire regarding demographic factors and to score their self-perceived levels of oral malodor. They responded whether or not they had symptoms related to xerostomia and gingival bleeding, and whether or not they had received periodontal treatment such as periodontal surgery, scaling, or root planing within the previous 3 months.

\subsection{Organoleptic Score Measurements}

To avoid any bias, the organoleptic score (OLS) was evaluated before making any other measurements. Scores were assigned by two trained judges who tested their ability to distinguish the severity of the oral malodor in a preliminary test to standardize their judging criteria. A plastic wall $(90 \mathrm{~cm} \times 120 \mathrm{~cm})$ with a central hole equipped with a Teflon tube (internal diameter $=$ $2.9 \mathrm{~cm}$, length $=10 \mathrm{~cm}$ ) was placed between the subject and the examiner. Subjects were instructed to close their mouth 1 min prior to sample collection and then to blow out the air inside their mouth through the tube for $5 \mathrm{~s}$. The exhaled air was scored by the examiner as described by Rosenberg et al. ${ }^{29,30}$ as follows: 0 , absence of odor; 1 , barely noticeable odor; 2 , slight odor; 3 , moderate odor; 4 , strong odor; and 5, extremely strong odor. In cases of measurement disagreement between the two examiners, a representative score was determined by consensus. There was a high interexaminer reliability for the OLS measurements made by the two judges $(k=0.88)$. Subjects with a score $>1$ were considered to have definite oral malodor in this study.

\subsection{Volatile Sulfur Compound Measurements}

The VSC concentrations in mouth air were measured using a portable gas chromatograph analyzer (OralChroma ${ }^{\mathrm{TM}}$, Abilit Corporation, Kanagawa, Japan) in accordance with the manufacturer's instructions. This device measures the concentrations of the following three VSCs in parts per billion: $\mathrm{H}_{2} \mathrm{~S}, \mathrm{CH}_{3} \mathrm{SH}$, and $\left(\mathrm{CH}_{3}\right)_{2} \mathrm{~S}$. The resulting chromatogram was reviewed to discard erroneous data due to known limitations of the device. ${ }^{31}$ After each subject closed their mouth and breathed nasally for $3 \mathrm{~min}$, the two-thirds of a disposable 1-ml plastic syringe were deeply inserted into the nearly closed mouth. During the sampling the subjects were instructed to refrain from inhaling or exhaling orally to prevent the tongue from contacting the syringe. After an air sample was collected, a $0.5-\mathrm{ml}$ aliquot was injected into the device. All of these measurements were performed by a single-trained examiner (Hyun-Kyung Yim).

\subsection{Fluorescence Image Acquisition}

Plaque fluorescence images were obtained using QLF-D (QLFD Biluminator ${ }^{\mathrm{TM}}$, Inspektor Research Systems, the Netherlands) and proprietary software (C2 version 1.0.0.7, Inspektor Research Systems) for the camera settings. The QLF-D Biluminator ${ }^{\mathrm{TM}}$, a device using QLF technology, uses a digital single-lens reflex (SLR) camera with blue and white LED lights with a peak wavelength of $405 \pm 20 \mathrm{~nm}$ (violet) and modified filter set (D007; Inspektor Research Systems, the Netherlands). This device captured the normal white-light images and fluorescence images of the tongue and floss with a "Live-View"enabled SLR camera (model 550D, Canon, Tokyo, Japan) using the following settings: shutter speed of $1 / 45 \mathrm{~s}$, aperture value of 3.2, and ISO 1600 (Fig. 1).

To ensure that the distance between the tongue and light source remained constant while capturing the whole tongue area, a subsidiary cylindrical ring was equipped with the camera tube, which enables the examiner to easily position a patient's face and tongue. The subject was instructed to extend his or her tongue out, and the examiner manually extended the tongue as much as possible to both minimize its movements during image acquisition and expose a maximum area of the tongue. ${ }^{32}$ The examiner fixed the end of the cylindrical ring on the patient's face and took the tongue images.

The interdental plaque was collected by flossing the area between the first and the second molar in each of the four quadrants. The sampling was omitted if any of the molars were missing from each quadrant. The floss was passed through the contact area and flossed below the gingival margin as deeply as possible, and supra- and subgingival plaque samples were collected simultaneously. The examiner flossed each side of the molar twice in the same way, with a total of four flossing procedures being performed. When removing the floss from the interdental area, the end part of the floss was passed by the contact point to avoid influencing the collected plaque on the middle part of the floss. All sampling procedures were performed by a single-trained examiner (Eun-Song Lee). Disposable floss (WE DEN, Kimpo, Korea) designed for use with a handle and with a constant working length was used. As soon as the collection of plaque samples was completed, 

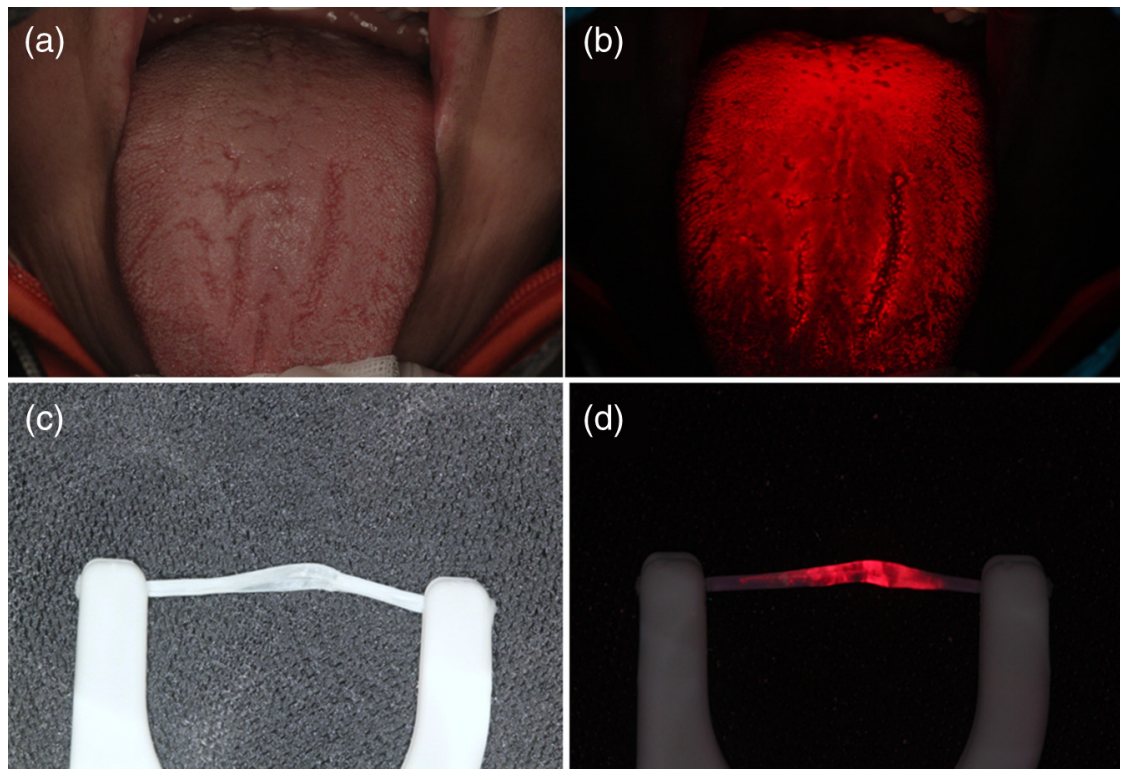

Fig. 1 Example images of tongue plaque (a), (b) and interdental plaque (c), (d) captured by QLF-D using white light (a), (b) and blue light (b), (d).

normal white-light images and sequential fluorescence images of the interdental plaque were obtained using QLF-D using the same settings used to obtain the tongue images.

\subsection{Image Analysis and Fluorescence Score Calculations}

The RF properties of the plaque in QLF-D images were quantified using image analysis software (Image-Pro Plus 6.0, Media Cybernetics, Rockville, Maryland). An area of interest (AOI) was drawn around the tongue boundary on the white-light image to calculate the total area of each tongue. The AOI from the normal image was imported into the fluorescence image, and then the intensity of RF and the area within the AOI for each image were determined. To account for differences in tongue size between individuals, the RF intensity value was obtained by calculating the mean ratio of the red and green intensities $(R / G$ ratio) of every pixel, and the RF area was derived as a percentage by calculating the ratio of the number of red-fluorescencing pixels to the total number of pixels within the AOI. ${ }^{32}$ The interdental plaque was analyzed to reflect the differences in the amounts of plaque collected from the flossed area. An AOI of the entire flossed area was drawn around the boundary of the flossed area in the fluorescence image. The red intensity $(R / G$ ratio) and fluorescent area (a percentage) within the flossed AOI were analyzed in the same way as for the whole tongue. By multiplying the fluorescence area and intensity of each type of plaque, the fluorescence score was calculated as follows: tongue plaque fluorescence (TPF) score $=$ $\mathrm{RF}$ intensity $(R / G$ ratio $) \times \mathrm{RF}$ area $(\%)$, and interdental plaque fluorescence (IPF) score $=\mathrm{RF}$ intensity $(R / G$ ratio $) \times \mathrm{RF}$ area $(\%)$. Four IPF scores at four sites for each subject were calculated and the IPF score for that individual represented the average of these four IPF scores. Finally, to obtain individual plaque scores that represented the total plaque (tongue and interdental) area, the CPF score was calculated by adding the two fluorescence scores (TPF and IPF scores) for each subject.

\subsection{Statistical Analysis}

The Kolmogorov-Smirnov test was performed to confirm the normality of all variables. To compare the subjects with and without definite oral malodor, $p$-values were calculated with the two-sample $t$-test and chi-squared tests. The median values of fluorescence variables from tongue, interdental plaque, and the $\mathrm{CPF}$ score were compared among three severity groups (none: OLS 0-1, slight-moderate: OLS 2-3, strong-severe: OLS 4-5) using a Mann-Whitney U-test with Bonferroni's adjustment. The relationships of oral malodor with TPF and IPF scores were assessed using Spearman correlation coefficients. A receiver operating characteristic (ROC) analysis was used to investigate the overall performance of fluorescence scores based on QLF-D in diagnosing oral malodor. With the OLS as a gold standard for the severity of oral malodor, the sensitivity and specificity values of the CPF score were calculated along with $95 \%$ confidence intervals. To assess the accuracy in detecting each diagnostic threshold of oral malodor, the area under the ROC curve (AUC) for the CPF score was calculated. The optimal cutoff values of CPF scores were determined based on the highest sum of sensitivity and specificity for each threshold. For all analyses, a 5\% significance cutoff was adopted and the data were analyzed using PASW Statistics software (version 18.0, SPSS, IBM Corporation, Somers, New York) and MedCalc ${ }^{\circledR}$ (version 8.1.1.0, MedCalc Software, Ostend, Belgium).

\section{Results}

About 99 patients included in the present study ranged in age from 19 to 66 years $(36.3 \pm 13.1$ years). The organoleptic evaluations revealed that $83.8 \%$ of the patients had definite oral malodor (OLS $=2$ to 5). The OLS score and VSC levels were significantly higher in the obvious-malodor group than in the no-malodor group ( $p<0.0001$, Table 1$)$. About $\sim 21 \%$ of the subjects had received periodontal treatment within the previous 3 months. About $\sim 39 \%$ of the subjects with definite oral malodor had symptoms of gingival bleeding, whereas this was present in $\sim 19 \%$ of the subjects without oral malodor 
Table 1 Characteristics and clinical parameters of the study population $(n=99)$.

\begin{tabular}{|c|c|c|c|}
\hline Parameter & $\begin{array}{l}\text { No obvious } \\
\text { oral malodor } \\
\text { OLS } 0,1\end{array}$ & $\begin{array}{l}\text { Obvious oral } \\
\text { malodor } \\
\text { OLS } \geq 2\end{array}$ & $\begin{array}{c}\text { Total } \\
p \text {-value }\end{array}$ \\
\hline Subject $(n / \%)$ & $16(16.2)$ & $83(83.8)$ & \\
\hline \multicolumn{4}{|l|}{ Gender $(n)$} \\
\hline Female & 8 & 48 & $56(56.6)$ \\
\hline Male & 8 & 35 & $43(43.4)$ \\
\hline Age $($ mean $\pm S D)$ & $26.3 \pm 5.4$ & $38.2 \pm 13.2$ & $\begin{array}{l}36.3 \pm 13.1 \\
P=0.004^{\mathrm{a}}\end{array}$ \\
\hline OLS (mean $\pm \mathrm{SD})$ & $0.8 \pm 0.5$ & $3.3 \pm 0.9$ & $\begin{array}{c}2.9 \pm 1.3 \\
P<0.0001^{\mathrm{a}}\end{array}$ \\
\hline 0 & 4 & & \\
\hline 1 & 12 & & \\
\hline 2 & & 15 & \\
\hline 3 & & 31 & \\
\hline 4 & & 31 & \\
\hline 5 & & 6 & \\
\hline $\begin{array}{l}\text { Total VSC level } \\
\text { (mean } \pm \text { SD) }\end{array}$ & $78.1 \pm 51.5$ & $425.5 \pm 526.8$ & $\begin{array}{r}369.4 \pm 499.1 \\
P<0.0001^{a}\end{array}$ \\
\hline \multirow[t]{2}{*}{$\begin{array}{l}\text { Self-reported severity } \\
\text { of oral malodor } \\
\text { (mean } \pm \text { SD) }\end{array}$} & $2.2 \pm 1.1$ & $3.0 \pm 1.1$ & $2.9 \pm 1.2$ \\
\hline & & & $P=0.02^{\mathrm{a}}$ \\
\hline \multicolumn{4}{|c|}{ Periodontal treatment $(n / \%)$} \\
\hline Yes & $4(25)$ & $15(18.1)$ & $P=0.524^{b}$ \\
\hline No & $12(75)$ & $68(81.9)$ & \\
\hline \multicolumn{4}{|l|}{ Bleeding $(n / \%)$} \\
\hline Yes & $3(18.7)$ & $32(38.6)$ & $P=0.096^{\mathrm{b}}$ \\
\hline No & $13(81.3)$ & $51(61.4)$ & \\
\hline \multicolumn{4}{|l|}{ Xerostomia $(n / \%)$} \\
\hline Yes & $5(31.2)$ & $31(37.3)$ & $P=0.646^{b}$ \\
\hline No & $11(68.8)$ & $52(62.7)$ & \\
\hline
\end{tabular}

Note: OLS, organoleptic score.

${ }^{\mathrm{a}} \boldsymbol{t}$-test.

${ }^{\mathrm{b}}$ chi-squared test.

(Table 1). The presence of oral malodor was not significantly associated with periodontal treatment, gingival bleeding, or the symptoms of xerostomia $(p>0.05)$.

The fluorescence variables and scores of each plaque for the three severity groups classified according to the OLS are listed in Table 2. The CPF score increased with the malodor level and significantly differed among the groups $(p<0.0001)$. The fluorescence intensity and integrated score for tongue plaque also differed significantly between the groups $(p<0.0001)$. Similarly, there was a significant difference between the strong-severe level group and the other groups for the fluorescence area and the integrated score of the interdental plaque $(p<0.0001)$.

$R / G$ values represent the ratios of red to green pixels in $\mathrm{RF}$ images of the tongue captured by the QLF-D.

Different letters within the same column indicate significant differences between malodor groups by Bonferroni's correction for multiple analysis at $\alpha=0.05$.

Also, all plaque fluorescence scores showed significant positive correlations with the OLS and total VSCs level (Table 3, Fig. 2). Among them, the CPF score displayed the strongest correlations with OLS $(r=0.64, p<0.01)$ and the total VSCs level $(r=0.54, p<0.01)$. The TPF score was correlated more strongly with the malodor level ( $r=0.51$ to 0.55$)$ than with the IPF score ( $r=0.38$ to 0.47 ). Also, the significant correlations were observed between the CPF scores and $\mathrm{H}_{2} \mathrm{~S}(r=0.59$, $p<0.01)$ and $\mathrm{CH}_{3} \mathrm{SH}(r=0.31, p<0.01)$ concentrations, while there was no significant correlations between the fluorescence scores and $\left(\mathrm{CH}_{3}\right)_{2} \mathrm{~S}$ concentrations $(r=0.01, p=0.42$, Table 3).

The sensitivity, specificity, and AUC values for the CPF scores regarding the OLS to assess the different severities of oral malodor are presented in Table 4 . The diagnostic accuracy of $\mathrm{CPF}$ scores for oral malodor was moderate to high (AUC $=0.77$ to 0.94 ) and increased with the threshold level. When using a CPF score of 155.7 for detecting subjects with and without definite oral malodor (OLS 0-1/2-5), the sensitivity was $90.7 \%$ and the specificity was $62.9 \%$.

\section{Discussion}

The main finding of the present study was that oral malodor could be assessed by quantifying fluorescence of oral biofilms, which are considered to be major etiological factors for oral malodor. The two bacterial biofilms targeted in this study comprise VSCs-producing anaerobic bacteria mainly associated with periodontal diseases, ${ }^{11,12}$ which means that it is valid and reliable to evaluate the characteristics of these biofilms when assessing malodor.

In terms of optical phenomenon of plaque autofluorescence, RF emission observed on QLF technology is proposed to be the result of excitation of endogenous porphyrins by the violet-blue light at a 405-nm wavelength. ${ }^{19,33,34}$ The porphyrin concentration is high in Gram-negative oral bacteria, which indicates the observed RF was produced by mature bacterial biofilms, and its intensity increased with the biofilm maturation and its pathogenicity. ${ }^{19,23}$ These previous findings support the results of the present study that the RF of two biofilms was significantly correlated with the severity of oral malodor. Given the associations and the diagnostic accuracy of the CPF score, the combined approach of plaque fluorescence could be used to evaluate the severity of oral malodor with increased accuracy. In addition, the present study confirmed the potential of an assessment method in objectively determining oral hygiene status as well as malodor levels, which is meaningful for both motivating patients to perform plaque control themselves and for clinical applications aimed at reducing oral malodor. 
Table 2 Median values of fluorescence variables for each type of plaque according to the severity of oral malodor.

\begin{tabular}{|c|c|c|c|c|c|}
\hline \multirow{2}{*}{\multicolumn{2}{|c|}{ Fluorescence variables $^{A}$}} & \multicolumn{3}{|c|}{ Malodor groups } & \multirow[b]{2}{*}{$p$-value } \\
\hline & & None (OLS 0-1) & Slight-moderate (OLS 2-3) & Strong-severe (OLS 4-5) & \\
\hline$N(\%)$ & & $16(16.2)$ & $46(46.5)$ & $37(37.4)$ & \\
\hline VSC level (ppb) & & $67(17 \text { to } 197)^{a}$ & $199(2 \text { to } 733)^{b}$ & $505(84 \text { to } 3620)^{\mathrm{C}}$ & $<0.000$ \\
\hline \multirow[t]{3}{*}{ Tongue plaque } & Intensity & $2.4(1.8 \text { to } 3.2)^{a}$ & $2.5(1.8 \text { to } 5.3)^{\mathrm{a}}$ & $2.9(2.2 \text { to } 6.9)^{\mathrm{b}}$ & $<0.000$ \\
\hline & Area & $57.5(35.6 \text { to } 98.4)^{\mathrm{a}}$ & $68.0(0.6 \text { to } 98.4)^{\mathrm{a}}$ & $83(45.7 \text { to } 99.1)^{b}$ & 0.008 \\
\hline & TPF score (Intensity $\times$ area) & $138.9(85.6 \text { to } 222.7)^{\mathrm{a}}$ & $171.1(1.3 \text { to } 268.8)^{\mathrm{a}}$ & $225.5(104.6 \text { to } 667.1)^{b}$ & $<0.000$ \\
\hline \multirow[t]{3}{*}{ Interdental plaque } & Intensity & $2.3(1.8 \text { to } 5.0)^{\mathrm{a}}$ & $2.5(1.6 \text { to } 5.8)^{\mathrm{a}}$ & $2.8(1.9 \text { to } 10.4)^{\mathrm{a}}$ & 0.05 \\
\hline & Area & $8.8(1.6 \text { to } 34.1)^{\mathrm{a}}$ & $16.7(0.53 \text { to } 42.2)^{\mathrm{a}}$ & $24.9(2.6 \text { to } 51.0)^{\mathrm{b}}$ & $<0.000$ \\
\hline & IPF score (intensity $\times$ area) & $17.6(3.6 \text { to } 169.3)^{\mathrm{a}}$ & $41.2(0.9 \text { to } 187.2)^{\mathrm{a}}$ & $81.3(7.2 \text { to } 392.9)^{\mathrm{b}}$ & $<0.000$ \\
\hline \multicolumn{2}{|c|}{ CPF score (TPF score + IPF score) } & $150.9(115.0 \text { to } 392.0)^{\mathrm{a}}$ & $213.4(42.5 \text { to } 387.0)^{b}$ & $294.9(117.6 \text { to } 766.3)^{\mathrm{c}}$ & $<0.000$ \\
\hline
\end{tabular}

Note: OLS, organoleptic score; TPF score, tongue plaque fluorescence score; IPF score, interdental plaque fluorescence score; CPF score, combined plaque fluorescence score calculated by summing up the value of tongue score and interdental plaque score.

AValues are the median (min-max).

Different letters within the same row indicate significant differences between groups by Bonferroni's correction for multiple analysis at $\alpha=0.05$.

The results obtained in the present study demonstrate that the newly developed CPF score was more strongly correlated with oral malodor than each individual plaque score (Table 3 ). This is consistent with previous findings suggesting that the sites that most commonly produce oral malodor-the tongue dorsum, interdental area, and gingival sulcus-are also the main anatomical sources of VSCs. ${ }^{5}$ These areas may constitute specific environments that are relatively free from saliva flushing and have low oxygen levels, thereby favoring more anaerobic species and their bacterial biofilms containing high proportions of anaerobic Gram-negative bacteria. ${ }^{35,36}$ Moreover, an association of plaque fluorescence with oral malodor is also supported by previous findings that the anaerobic species within the biofilms can actively produce malodorous VSCs and short-chain organic acids via metabolic interactions. ${ }^{13}$ For these reasons, the assessment of bacterial samples obtained from the tongue and interdental sites has been used to diagnose oral malodor, such as using the benzoyl-DL-arginine-naphthylamide (BANA) test quantifying bacterial activity and the polymerase chain reaction. In addition, it is well known that oral malodor is induced by interactions between multiple species of bacteria, which support the measurement of fluorescence resulting from the metabolic activity of all bacteria for assessing oral malodor.

This is the first study to have visualized and quantified the amount of interdental plaque using an objective detection method. To assess the pathogenicity of interdental plaque, which is difficult to detect, it is imperative to collect plaque samples while preserving their mechanical and biochemical properties. Therefore, the interdental plaque in individual patients was sampled by flossing using the same method with standardized floss, and the accumulation status of the interdental plaque on the floss was quantified based on the fluorescence intensity and area. As the tendency for RF varied between the participants, it showed variations in the fluorescence intensity and distribution patterns (Fig. 1), we have proposed the use of an individual fluorescence score obtained by multiplying two fluorescence

Table 3 Correlation coefficients between oral malodor and fluorescence variables of the plaques.

Plaque fluorescence score

\begin{tabular}{|c|c|c|c|c|c|}
\hline & \multirow[b]{2}{*}{ OLS } & \multirow[b]{2}{*}{ Total VSC level } & \\
\hline & & & TPF score & IPF score & CPF score (TPF score + IPF score) \\
\hline OLS & & $0.72^{\star *}$ & $0.55^{\star *}$ & $0.47^{\star *}$ & $0.64^{\star *}$ \\
\hline Total VSC level (ppb) & $0.72^{\star \star}$ & & $0.51^{\star *}$ & $0.38^{\star *}$ & $0.54^{\star *}$ \\
\hline $\mathrm{H}_{2} \mathrm{~S}$ & $0.62^{\star \star}$ & $0.83^{\star \star}$ & $0.52^{\star \star}$ & $0.49^{* *}$ & $0.59^{* *}$ \\
\hline $\mathrm{CH}_{3} \mathrm{SH}$ & $0.43^{\star \star}$ & $0.61^{\star *}$ & $0.32^{\star \star}$ & $0.29^{*}$ & $0.31^{*}$ \\
\hline$\left(\mathrm{CH}_{3}\right)_{2} \mathrm{~S}$ & 0.2 & $0.45^{\star *}$ & 0.05 & -0.02 & 0.01 \\
\hline
\end{tabular}

Note: OLS, organoleptic score; TPF score, tongue plaque fluorescence score; IPF score, interdental plaque fluorescence score; CPF score, combined plaque fluorescence score.

Spearman correlation analysis, ${ }^{\star} P<0.05,{ }^{* \star} P<0.01$. 


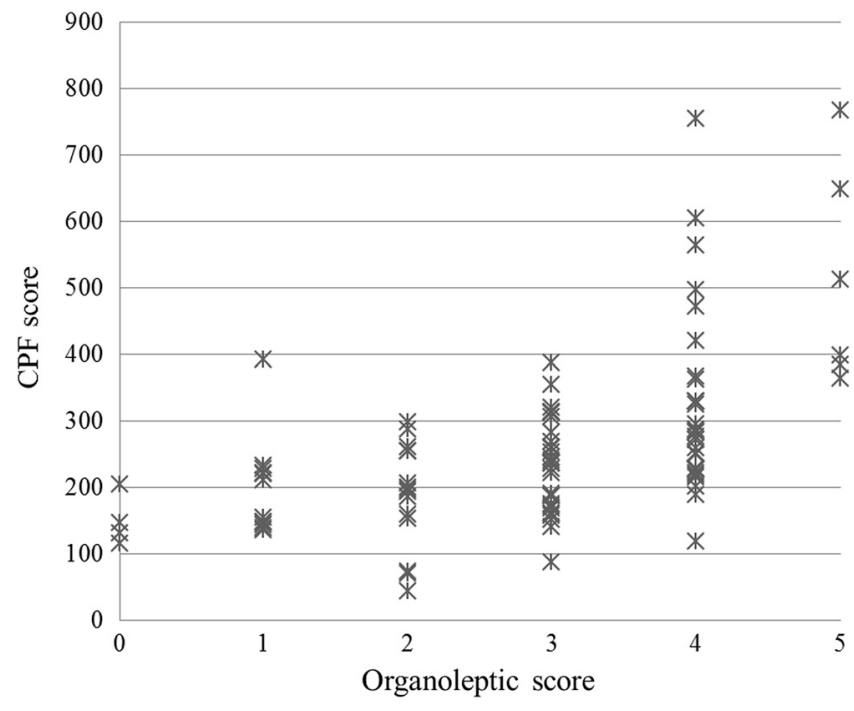

Fig. 2 Scatter plots of the correlations between the OLS and CPF score.

properties. In terms of the interdental plaque being a predictor for gingival inflammation and the periodontal status in an individual, the findings of the present study suggest that the method using QLF technology would be useful for early detection of plaque-related disease.

In the present study, the IPF score was significantly correlated with the OLS and the VSC levels, especially of $\mathrm{CH}_{3} \mathrm{SH}$ and $\mathrm{H}_{2} \mathrm{~S}$ (Table 3), which indicates that evaluating the amount of interdental plaque could be useful for diagnosing oral malodor. Emphasizing the role of interdental plaque as a status indicator for oral hygiene and gingival health, the present findings are consistent with previous reports of oral malodor being primarily associated with the tongue coating and gingival inflammation. ${ }^{37}$ Previous researchers found that the interdental plaque and subgingival plaque of patients with oral malodor contain significantly higher proportions of anaerobic bacteria that produce VSCs, and that patients with periodontal pocket and inflamed gingiva showed significantly higher concentrations of VSCs. ${ }^{13,38,39}$ Moreover, there was a strong relationship between plaque-induced periodontal disease and the severity of oral malodor, since periodontal pathogens such as

Table 4 AUC and optimum sensitivity, specificity, and cutoff values for the CPF score at each diagnostic threshold quantified using the OLS.

\begin{tabular}{cccccccc}
\multirow{2}{*}{$\begin{array}{c}\text { Oral malodor } \\
\text { criteria }\end{array}$} & AUC & $95 \%$ Cl & $p$-value & & & & \\
\cline { 2 - 6 } & SE (\%) & SP (\%) & Cut-off \\
\hline OLS 0,1/2-5 & 0.77 & 0.68 to 0.85 & $<0.0001$ & 90.7 & 62.9 & 155.7 \\
OLS 0-2/3-5 & 0.79 & 0.70 to 0.88 & $<0.0001$ & 77.5 & 66.6 & 213.4 \\
OLS 0-3/4,5 & 0.79 & 0.70 to 0.86 & $<0.0001$ & 75.7 & 68.2 & 246.8 \\
OLS 0-4/5 & 0.94 & 0.90 to 0.99 & $<0.0001$ & 100.0 & 89.6 & 362.6 \\
\hline
\end{tabular}

Note: AUC, area under the ROC curve; $\mathrm{Cl}$, confidence interval; SE, sensitivity; SP, specificity.
Porphyromonas gingivalis, Prevotella intermedia, Tannerella denticola, and Veillonella alcalescens in the subgingival area can produce malodorous compounds. This further suggests that assessment of interdental plaque can be used to evaluate oral malodor as a useful predictor of the periodontal status of an individual patient.

It is presumed that the two biofilms have similar bacterial compositions and, in particular, share the presence of redfluorescencing bacteria, confirming the significant correlation between the two plaque fluorescence scores in this study. Based on previous studies, the microbes identified in the tongue plaque are almost the same as those found in the subgingival plaque, ${ }^{11,12,40-42}$ and the tongue-coating volume is significantly correlated with the percentage area of bleeding-on-probing sites and the severity of periodontal disease. ${ }^{37,41}$ We speculate that there is a relationship between the microflora of the tongue plaque and of the interdental plaque, where the accumulation of anaerobic bacteria in the biofilms can affect the production of oral malodor.

The present study has revealed the potential of QLF technology for detecting bacterial biofilms in diagnosing oral malodor by objectively assessing the comprehensive properties of pathogenic biofilms that are closely involved in the generation of oral malodor. The clinical use of QLF technology may make it possible for patients to check their own oral hygiene status and provide a quantitative numerical value that accurately reflects the severity of oral malodor. However, some improvements are necessary to the convenience and accuracy when applying this method in clinical applications. For example, analysis software based on algorithms for calculating the combined fluorescence score found in this study needs to be developed, and additional equipment is needed to adequately fix the tongue position when capturing the QLF images.

\section{Conclusions}

It can be concluded that the red autofluorescence from tongue and interdental plaque detected using QLF technology was significantly correlated with the level of oral malodor. Therefore, the plaque fluorescence score which represents comprehensive fluorescence properties of oral biofilms could be used to quantify the level of oral malodor and to aid its diagnosis.

\section{Acknowledgments}

This research was supported by the Basic Science Research Program through the National Research Foundation of Korea (NRF), funded by the Ministry of Education (2013R1A1A2062505), and supported in part by the Yonsei University Future-leading Research Initiative of 2014 (201422-0171).

\section{References}

1. H. Miyazaki et al., "Correlation between volatile sulphur compounds and certain oral health measurements in the general population," J. Periodontol. 66(8), 679-684 (1995).

2. B. Vandekerckhove et al., "Clinical reliability of non-organoleptic oral malodour measurements," J. Clin. Periodontol. 36(11), 964-969 (2009).

3. J. Tonzetich, "Production and origin of oral malodor: a review of mechanisms and methods of analysis," J. Periodontol. 48(1), 13-20 (1977).

4. E. H. De Boever and W. J. Loesche, "Assessing the contribution of anaerobic microflora of the tongue to oral malodor," J. Am. Dent. Assoc. 126(10), 1384-1393 (1995). 
5. M. Rosenberg, "Clinical assessment of bad breath: current concepts," J. Am. Dent. Assoc. 127(4), 475-482 (1996).

6. M. Faveri et al., "A cross-over study on the effect of various therapeutic approaches to morning breath odour," J. Clin. Periodontol. 33(8), 555-560 (2006).

7. Y. Nakano, M. Yoshimura, and T. Koga, "Correlation between oral malodor and periodontal bacteria," Microbes Infect./Inst. Pasteur 4(6), 679-683 (2002).

8. B. U. Aylikci and H. Colak, "Halitosis: from diagnosis to management," J. Nat. Sci. Biol. Med. 4(1), 14-23 (2013).

9. A. Bosy et al., "Relationship of oral malodor to periodontitis: evidence of independence in discrete subpopulations," J. Periodontol. 65(1), 37-46 (1994).

10. T. Shimizu, T. Ueda, and K. Sakurai, "New method for evaluation of tongue-coating status," J. Oral Rehabil. 34(6), 442-447 (2007).

11. T. Yasukawa, M. Ohmori, and S. Sato, "The relationship between physiologic halitosis and periodontopathic bacteria of the tongue and gingival sulcus," Odontology 98(1), 44-51 (2010).

12. M. Matsui et al., "Effects of tongue cleaning on bacterial flora in tongue coating and dental plaque: a crossover study," BMC Oral Health 14, 4 (2014).

13. C. E. Kazor et al., "Diversity of bacterial populations on the tongue dorsa of patients with halitosis and healthy patients," J. Clin. Microbiol. 41(2), 558-563 (2003).

14. T. Lundgren et al., "Evaluation of tongue coating indices," Oral Dis. 13(2), 177-180 (2007).

15. M. Tanaka et al., "Reliability of clinical parameters for predicting the outcome of oral malodor treatment," J. Dent. Res. 82(7), 518-522 (2003).

16. K. Yaegaki and K. Sanada, "Volatile sulfur compounds in mouth air from clinically healthy subjects and patients with periodontal disease," J. Periodontal Res. 27(4 Pt 1), 233-238 (1992).

17. S. Saad and J. Greenman, "Tongue biofilm areal density and tongue coating index," J. Breath Res. 2(1), 017008 (2008).

18. C. M. Volgenant et al., "Effect of metalloporphyrins on red autofluorescence from oral bacteria," J. Eur. Oral Sci. 121(3 Pt 1), 156-161 (2013).

19. K. Konig, G. Flemming, and R. Hibst, "Laser-induced autofluorescence spectroscopy of dental caries," Cell. Mol. Biol. (Noisy-le-grand) 44(8), 1293-1300 (1998).

20. L. Coulthwaite et al., "The microbiological origin of fluorescence observed in plaque on dentures during QLF analysis," Caries Res. 40(2), 112-116 (2006).

21. R. Z. Thomas et al., "Bacterial composition and red fluorescence of plaque in relation to primary and secondary caries next to composite: an in situ study," Oral Microbiol. Immunol. 23(1), 7-13 (2008).

22. A. M. Lennon et al., "The ability of selected oral microorganisms to emit red fluorescence," Caries Res. 40(1), 2-5 (2006).

23. Y. S. Kim et al., "Monitoring the maturation process of a dental microcosm biofilm using the quantitative light-induced fluorescence-digital (QLF-D)," J. Dent. 42(6), 691-696 (2014).

24. M. R. Alammari et al., "Quantitative light-induced fluorescence (QLF): a tool for early occlusal dental caries detection and supporting decision making in vivo," J. Dent. 41(2), 127-132 (2013).

25. E. S. Lee et al., "Association between the cariogenicity of a dental microcosm biofilm and its red fluorescence detected by quantitative light-induced fluorescence-digital (QLF-D)," J. Dent. 41(12), 12641270 (2013).
26. S. Y. Han et al., "Validity and reliability of autofluorescence-based quantification method of dental plaque," Photodiagn. Photodyn. Ther. 12(4), 587-591. (2015).

27. E. S. Lee et al., "Clinical assessment of oral malodor using autofluorescence of tongue coating," Photodiagn. Photodyn. Ther. 13, 323-329 (2016).

28. A. Flahault, M. Cadilhac, and G. Thomas, "Sample size calculation should be performed for design accuracy in diagnostic test studies," J. Clin. Epidemiol. 58(8), 859-862 (2005).

29. M. Rosenberg et al., "Halitosis measurement by an industrial sulphide monitor," J. Periodontol. 62(8), 487-489 (1991).

30. M. Rosenberg et al., "Reproducibility and sensitivity of oral malodor measurements with a portable sulphide monitor," J. Dent. Res. 70(11), 1436-1440 (1991).

31. A. Tangerman and E. G. Winkel, "The portable gas chromatograph OralChroma: a method of choice to detect oral and extra-oral halitosis," J. Breath Res. 2(1), 017010 (2008).

32. E. S. Lee et al., "Clinical assessment of oral malodor using autofluorescence of tongue coating," Photodiagn. Photodyn. Ther. 13, 323-329. (2015).

33. M. H. van der Veen et al., "Red autofluorescence of dental plaque bacteria," Caries Res. 40(6), 542-545 (2006).

34. G. Felix Gomez, G. J. Eckert, and A. Ferreira Zandona, "Orange/red fluorescence of active caries by retrospective quantitative light-induced fluorescence image analysis," Caries Res. 50(3), 295-302 (2016).

35. M. Quirynen, "Management of oral malodour," J. Clin. Periodontol. 30(Suppl 5), 17-18 (2003).

36. A. C. Donaldson et al., "Microbiological culture analysis of the tongue anaerobic microflora in subjects with and without halitosis," Oral Dis. 11(Suppl 1), 61-63 (2005).

37. M. Morita and H. L. Wang, "Relationship between sulcular sulfide level and oral malodor in subjects with periodontal disease," J. Periodontol. 72(1), 79-84 (2001).

38. S. Mantilla Gomez et al., "Tongue coating and salivary bacterial counts in healthy/gingivitis subjects and periodontitis patients," J. Clin. Periodontol. 28(10), 970-978 (2001).

39. C. Calil et al., "The relationship between volatile sulphur compounds, tongue coating and periodontal disease," Int. J. Dent. Hyg. 7(4), 251-255 (2009).

40. M. Tanaka et al., "Contribution of periodontal pathogens on tongue dorsa analyzed with real-time PCR to oral malodor," Microbes Infect./Inst. Pasteur 6(12), 1078-1083 (2004).

41. M. Morita and H. L. Wang, "Association between oral malodor and adult periodontitis: a review," J. Clin. Periodontol. 28(9), 813-819 (2001).

42. M. Kishi et al., "Prediction of periodontopathic bacteria in dental plaque of periodontal healthy subjects by measurement of volatile sulfur compounds in mouth air," Arch. Oral Biol. 58(3), 324-330 (2013).

Eun-Song Lee received her BS degree in dental hygiene from Yonsei University in 2010, and her MS and PhD degrees in preventive dentistry from Yonsei University College of Dentistry in 2015. She is a research assistant professor at Yonsei University College of Dentistry. Her research interests include oral biofilm, oral microbiome, and optical detection and diagnosis for oral disease.

Biographies for the other authors are not available. 